Pub. Mat. $U A B$

$N^{\circ} 20$ Set. 1980

Actes VII JMHL

\title{
TRIPLES ASOCIADOS
}

J.M. Barja Pérez, J.I. Freire Nistal

Dpto. de Algebra y Fundamentos

Universidad de Santiago de Compostela

Abstract Two triple structures on a ? nctor $T ; T^{1}=(T, \eta, \mu)$ y $\mathbb{T}^{*}=$ $=\left(T, \eta^{*}, \mu^{*}\right)$ are to be called associated, $E\left(T_{,} T^{\star}\right)$, if $\mu^{*} \mu^{*} T^{*}=\mu^{\star} \cdot T_{\mu}$ $E\left(\mathbb{T}, \mathbb{T}^{*}\right)$ it is characterized by:existence of the superior degree triple structures, lifts to the respective algebras and Kleisli categories and, in this latter case, the existence of a pair of adjoint functors between them.

Comunicación:

Dos triples $\mathbb{T}=(T, \eta, \mu)$ y $\mathbb{T}^{\star}=\left(T, \eta^{\star}, \mu^{\star}\right)$ sobre un mismo funtor $\mathrm{T}$ en una categoria.K, se liaman asociados $E\left(T, \mathrm{~T}^{*}\right)$, si se verifica la ley del"triple producto": $\mu^{*} \mu^{\star} \mathrm{T}=\mu^{\star} \cdot \mathrm{T} \mu$.

Si $\mathrm{f}: \mathrm{T} \longrightarrow \mathrm{T}, \mathrm{g}: \mathrm{T} \longrightarrow \mathrm{T}$ son transformaciones naturales, ta les que $f \cdot \eta^{\prime} \eta^{*}, g \cdot \eta^{*}=\eta, y$ verifican la "ley de naturalidad": $\mu \cdot \mathrm{T}_{\mu} \cdot \mathrm{gT}^{2}=\mu^{*} \cdot \mathrm{T}_{\mu^{*}} \cdot \mathrm{T}^{2} \mathrm{f}$, entonces $\mathrm{E}\left(\mathrm{T}, \mathrm{TH}^{*}\right) \mathrm{Y}$, reciprocamente, $f=\mu \cdot r_{i}^{*}$ y $g=\mu^{*} \cdot T^{\prime}$ son. Ios bnicos morfismos de triples que cumplen la ley de naturalidad.

Los triples T y $T^{*}$ se llaman trivialmente asociados $s i$ $f 6 \mathrm{~g}$ son isomorfismos. Si $E\left(\mathrm{TP}^{*}\right)$, entonces $f$ isomorfismo $\Leftrightarrow$ $\Leftrightarrow E\left(\mathbb{T}^{\star}, \mathbb{T}\right) \Leftrightarrow \mathrm{g}$ isomorfismo.

Tambiên, $E\left(\mathbb{T}, \mathbb{T}^{\star}\right) \Leftrightarrow\left(T, T, \eta \eta^{\star}, \mu^{\star *} \mu T\right)$ es un triple de gra do superior $\Leftrightarrow\left(T, T, \eta n^{*}, \mu \cdot T_{\mu}^{*}\right)$ es un triple de grado superior. (Triple de grado superior, de grado 3 , en sentido de Maranda $17\}$

$E$ (T, $\mathbb{T}^{*}$ ) es equivalente a que la elevacion a las categorias de slgebras $\mathrm{Sg}: K^{T} \longrightarrow K^{T^{*}}$, verifique: $\mathrm{F}^{*}=\mathrm{Sg} \cdot \mathrm{F}^{\mathrm{T}}, \mathrm{Y}$ asimis- 
mo que SKf: $K_{\text {TI }} \longrightarrow K_{\text {TI* }}$, elevación a las categorias de álgebras de Kleisli, verifique: $u^{\mathbb{T}}=u^{T^{*}}$. Skf .

Se obtiene asl el diagrama conmutativo:

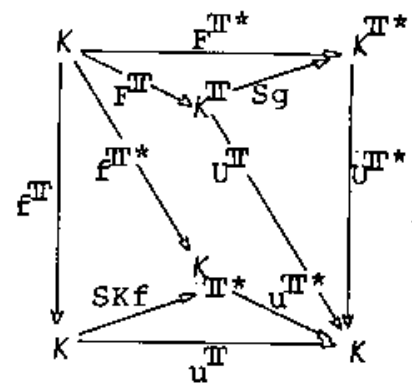

Si $T$ y $\mathbb{T}^{*}$ son asociados,para los monomorfismos de triples

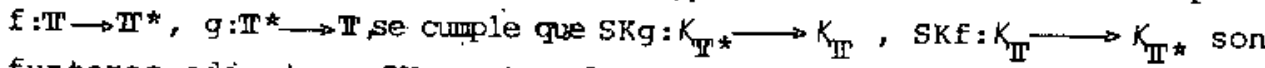
funtores adjuntos, SKg $\longrightarrow$ Skf. Reciprocamente, si $\mathbb{W}_{\mathbb{I}}$ y $\mathbb{T}^{\prime}$ son triples en $K, f: \mathbb{R} \longrightarrow \mathbb{T}^{\prime}, g: \mathbb{T}^{\prime} \longrightarrow \mathbb{T}$ morfísmos de triples tales que SKg - SKf, entonces existe un triple 'pt* isomorfo a Th', asociado con $\mathbb{T}$.

Si $E(A, B)$ es un klgebra de Kleisli (|2|) sobre el conjun to $E$, entonces $T^{\prime}=\left(E x-, a x-, \cdot x^{-}\right), T^{*}=\left(E x-, b x-, \star x^{-}\right)$son triples asociados en la categoría de conjuntos;y as 5 , existen triples no trivialmente asociados. Reciprocamente, si $E\left(\mathbb{T}, \mathbb{T}^{*}\right)$, en el con junto de operaciones unarias de $T$ queda definida una estructura de algebra de Kleisli.

Para A anillo conmutativo con 1 y $(E, \cdot, a, *, b)$ algebra de Kleisli no trivial en Conjuntos, las algebras de polinomios $A[E]$ correspondientes a los monoides del slgebra de Kleisli, de terminan triples asociados no triviales en al categorla de A-mó dulos.

El original completo de este trabajo aparecera publicado en Alxebra 26. Depto. Algebra y Fund. Santiago.

Bibliografia

|1| Barja Perez, J.M. Teoremas de Morita para triples en categorías cerradas. Alxebra 20.'(1979). Dpto. Algebra y Fund. Santiago.

|2| Barja Perez, J.M.;G-Rodeja F.E. Algebras de Kleisli. (En esta publicacion)

|3| Caruncho Castro, J.R. Teorfa de Triples. Alxebra 5 (1971) Dpto.Algebra y Fund. Santiago. 
14| Freire Nistal,J.L.Propiedades Universales en triples de grado superior. Alxebra 11 (1972) Dpto. Algebra y Fund. Santiago.

|5| Manes, E.G. Algebraic Theories. Springer (1976).

|6| Maranda, J.M. On fundamental construction and adjoint functors.Bull.Can.Math v9 (1966) 581-591.

17| Maranda,J.M. Constructions fondamentales de aegre superieur.J.Reine Angew Math.243(1970) 1-16. 